\title{
Successful Management of Jaundice in a Dog using Therapeutic Blood Transfusion
}

\author{
Tarun Kumar ${ }^{1 *}$, Kanchan Arya ${ }^{2}$, Pardeep Dhillon², Jai Bhagwan², \\ Maneesh Sharma ${ }^{1}$, Deepika Kumari ${ }^{2}$, Divya Agnihotri ${ }^{1}$ and V. K. Jain ${ }^{1}$ \\ ${ }^{1}$ Veterinary Clinical Complex, ${ }^{2}$ Department of Veterinary Medicine, \\ Lala Lajpat Rai University of Veterinary and Animal Sciences, \\ Hisar-125004 (Haryana), India \\ *Corresponding author
}

\section{A B S T R A C T}

\section{Keywords}

Dog, Jaundice, Anemia, Blood Transfusion

Article Info

Accepted:

05 June 2020 Available Online: 10 July 2020
An adult 1.5-year old male Pitbull dog was presented to the Department of veterinary clinical complex, LUVAS, Hisar, Haryana, with a history of anorexia, high fever, deep yellow colored urine and itching on ventral abdomen for one month. Clinical examination showed pale mucus membranes with increased respiration rate, however, electrocardiogram (ECG) was found normal. The hematological findings showed severe microcytic hypochromic anemia, neutrophilic leukocytosis, marked increase in total bilirubin values suggestive of jaundice. Bloodsmear examination was found negative for hemoprotozoan. The animal was treated with a combination therapy comprising of a single dose of Imidocarb dipropinate $(5.5 \mathrm{mg} / \mathrm{kg}$, b.wt, SC) and Ceftrixone-Tazobactum (25 $\mathrm{mg} / \mathrm{kg}$, b.wt, IM) for 5 days. Blood transfusion was conducted on the second day to correct severe anemia Supportive treatment included antiemetics, hepatoprotectants, hematinic, multivitamins, amino acids and fluid therapy. Reevaluation of various blood parameters after 7 days of treatment revealed marked improvement.

\section{Introduction}

Jaundice is a yellowish discoloration of the skin and mucous membranes caused by hyperbilirubinemia. Hyperbilirubinemia can occur due to prehepatic (usually hemolysis), posthepatic (due to obstructive or functional cholestasis), or hepatic causes. Hyperbilirubinemia in hepatic disease is caused by decreased uptake of unconjugated bilirubin and/or decreased conjugation by the hepatocytes (Sevelius, 1995) therefore, hyperbilirubinemia is usually associated with a severely impaired hepatic function.

Prehepatic jaundice is usually caused by the release of hemoglobin associated with $\mathrm{RBC}$ lysis. High plasma levels of unconjugated (free) bilirubin are derived from massive hemoglobin breakdown. 
Patients with prehepatic jaundice are therefore invariably severely anemic. Ongoing hemolysis of RBC's in sufficient magnitude causes persistent jaundice that would cause death due to anemia. Various causes of anemia includes infection or acute/chronic hemorrhage due to hemoprotozoan infections, bleeding disorders such as thrombocytopenia or coagulopathies drugs, and toxic plants such as warfarin poisonings (Bhikane and Kawitker, 2002).

The predominant clinical signs of jaundice are anemia, weakness, pale mucous membranes, tachycardia and tachypnea. Severe intravascular hemolysis may cause hemoglobinemia and hemoglobinuria (darked coloured urine).

Transfusion of whole blood or its constituents (red blood cells, white blood cells, plasma, platelets, and different clotting factors) is very useful to replace the lost components of blood, as in case of severe anemia, acute hemorrhage, coagulation disorders and thrombocytopenia. Blood transfusion is a process under which intravenous injection of blood products is carried out from donor to the recipient organism of the same species. Blood transfusion has been used in emergency as a life-saving process, since many years in animal and human medicine (Davidow, 2013). In the year 1665, Richard Lower reported the first successful blood transfusion in animals whereby blood was withdrawn from one dog and replaced with blood from another dog. (Lower,1665).

With the help of latest techniques and equipments after 1950, blood transfusion became more popular in veterinary medicine (Cotter,1991). Blood transfusion depends on multiple factors like the type of anemia, blood group, blood parameters, animal size and blood products to be administered (Lanevschi and Wardrop, 2001).
In clinical therapy, it is a common procedure, but substantial risks are involved because of living cell transfusion. US blood shield laws described blood transfusion as an "unavoidably, unsafe, and inherently dangerous" procedure (Zuck,1990).

Cats are more prone to its fatal effects as compared to dogs. The immediate adverse and potential transfusion reactions are urticaria, erythema or pruritus, vomiting, vocalization, pyrexia, dyspnea, tachypnea, coughing, tachycardia or bradycardia, tremors or convulsions, shock, and cardiopulmonary arrest. If there is any kind of adverse reaction, the transfusion should be stopped and treatment of adverse reaction should start immediately. The present case study reports successful therapeutic management of jaundice and anemia in a dog primarily with single blood transfusion along with other supportive treatment.

\section{Materials and Methods}

The present study was conducted in the department of veterinary clinical complex (VCC), LUVAS, Hisar, Haryana.

\section{Clinical history}

A 1.5-year male Pit bull weighing $25 \mathrm{~kg}$ was presented, with a history of deep yellow colored urine, fever, inappetence and itching on ventral abdomen along with decreased water intake for one month. In addition, there was also a history of incomplete deworming and vaccination. Dog was severely dehydrated and was lying in lateral recumbency at the time of presentation.

\section{Clinical and laboratory examination}

On clinical examination, the dog was having body temperature of $99.8^{0} \mathrm{~F}$, pale mucous membrane with increased respiration rate. 
The heart functioning of the dog was found to be normal by electrocardiogram (ECG) report. Blood samples were collected in EDTA containing vials for complete blood count (CBC) and Giemsa staining method for examination of haemoprotozoan infection. Serum analysis was done using Fully automated Random Access Clinical Chemistry Analyzer (EM 200 ErbaMannheim - Germany) for estimation of different biochemical parameters using kits procured from Transasia Biomedical Limited. Urine was collected aseptically using analysis urinary catheter and urinary analysis was performed using automated urinary strip analyzer (Laura Smart, Transaisa).

\section{Blood collection}

\section{Selection and screening of donor animals}

The donor dog selected for the blood donation was 1.5 years of age, weighing $21.8 \mathrm{~kg}$ and was of the same breed. The donor was found clinically healthy and was maintaining normal feed and water intake. Complete blood count and blood smear examination was performed for the donor dog and was interpreted as normal.

\section{Collection of blood from the donor}

Citrate Phosphate Dextrose Adenine (CPDA) bag of $350 \mathrm{ml}$ capacity was used for blood collection. Before blood collection, the donor was placed in the lateral recumbency and the jugular area of the dog was shaved and disinfected with spirit swab. The pressure was applied at the thoracic inlet with one hand to raise vein and with another hand, venipuncture was done to collect the blood directly in the bag with mild shaking during collection (Fig 1). Blood collection was completed within 15 minutes. Approximately $250 \mathrm{ml}$ of blood was collected from the donor (10-15 ml/kg blood of donor).

\section{Cross-matching}

Cross-matching of donor and recipient blood was done by the major and minor crossmatching method (Weiss and Wardrop, 2011). Blood from the donor as well as the recipient was collected in EDTA vials. Blood was centrifuged and separation of plasma from the RBCs was done in $1.5 \mathrm{ml}$ tubes. $200 \mathrm{ul}$ of RBCs were taken in a centrifuge tube and 4.8 $\mathrm{ml}$ of $0.9 \%$ NSS was added. Mixture was centrifuged for one minute after proper mixing. The process was repeated three times removing the supernatant every time. The final resuspension of the cells was done in NSS to make a $2 \%$ to $4 \%$ RBC solution $(0.2$ $\mathrm{ml}$ of blood in $4.8 \mathrm{ml}$ of saline gives a $4 \%$ solution). Labeling of the tubes was done to make the following mixtures: Major crossmatch (2 drops of recipient serum with one drop of donor RBC suspension), Minor crossmatch (1 drop of recipient RBC suspension with 2 drops of donor serum). Control (1 drop of recipient RBC suspension with 1 drop of recipient serum). All 3 tubes were incubated for 15 to 30 minutes at $37^{\circ} \mathrm{C}$ and then centrifuged for 15 seconds. The supernatant was examined for hemolysis or hemagglutination grossly as well as microscopically. Clumping of RBCs was taken as incompatible match whereas individual RBCs were taken as compatible.

\section{Administration of blood}

Before transfusing blood into the recipient $\mathrm{dog}$, all the risk of undergoing process was advocated to the owner. The injection of dexamethasone @ $0.25 \mathrm{mg} / \mathrm{kg}$ and pheniramine maleate @ $0.5 \mathrm{mg} / \mathrm{kg}$ were administered 15-20 minutes before initiating transfusion. Blood was given IV through cephalic vein via a blood administration set with a micropore filter to reduce the risk of entry of microthrombi into the general circulation and to give an estimate of 
calculated dose (Fig 2). The rate of transfusion was $0.25 \mathrm{ml} / \mathrm{kg}$ bwt. for the first 15 minutes to check any adverse reactions and then gradually increased to $2-10 \mathrm{ml} / \mathrm{kg} / \mathrm{hr}$ (Harrell and Kristensen, 1995). The transfusion was completed within 4 hours of collection of blood to minimize the risk of bacterial contamination.

\section{Monitoring and management of transfusion reaction}

Constant monitoring of the patient was done with the help of Multi-parameter patient monitor (Meditech Co. Ltd) during transfusion. Patient was critically monitored for the first 20 minutes for any common adverse reactions like acute hemolytic reaction, anaphylactic or allergic reactions etc. and after that vital parameters including body temperature, heart rate, respiration rate and oxygen saturation ( $\mathrm{SpO} 2)$ were monitored after every 20 minute still whole blood transfusion process.

\section{Results and Discussion}

Hematological examination revealed severe microcytic hypochromic anemia with neutrophilic leucocytosis (Table 1). The platelet count was in the normal range (2 lakh/cu.mm) with drastically increased liverspecific enzymes (AST and ALT).Total bilirubin, direct and indirect bilirubin values were increased significantly, suggestive of jaundice. Hemolytic anemia either due to hemoprotozon infection or immune immediate hemolysis was the tentative diagnosis. Although Geimsa staining didn't revealed any blood protozoan in the blood smear examination, but history of tick infestation and fever along with jaundice pointed out to be a case of hemoprotozoan infection with comorbidity of jaundice and anemia. Effect of blood transfusion on physiological parameters was evaluated
(Table 2) and no significant difference in temperature $(\mathrm{p}=0.50)$, pulse rate $(\mathrm{p}=0.48)$ and respiration rate $(\mathrm{p}=0.47)$ before and during blood transfusion was observed. Heart rate was 117 beats/minute before the start of transfusion and was almost stable after 40 minutes of transfusion ranging from 108 beats/minute to 104 beats/minute with no significant difference $(\mathrm{p}=0.55)$ in heart rate over the time. Hemoglobin saturation of oxygen (Spo2) was $65 \mathrm{mmHg}$ before the start of transfusion and increases drastically just after 20 minutes and reached at $94 \mathrm{mmHg}$. Later on, Spo2 got stabilized and ranged between $66 \mathrm{mmHg}$ to $84 \mathrm{mmHg}$ during the whole transfusion with no significant difference $(p=0.45)$ in Spo2 over the time. Measured hematobiochemical parameters pre and post blood transfusion was summarized in Table 1. The hemoglobin concentration before transfusion was $2.8 \mathrm{~g} \%$, which got increased to $6.1 \mathrm{~g} \%$ post blood transfusion. Packed cell volume too increased from $12 \%$ to $20 \%$ after transfusion (Chakrabarti, 2006).

\section{Treatment}

The dog was admitted and treated for 5 days by administering antibiotic CeftrixoneTazobactum @ 25mg/kg body weight along with parenteral supportive treatment including multivitamins. Blood transfusion was started immediately keeping in view the emergency of the case. Only one transfusion was done. Although patient was negative for hemoprotozoan on blood smear even then Imidocarb Dipropinate (Imizol®) @ of 5.5 $\mathrm{mg} / \mathrm{kg}$ body weight was given subcutaneously as single shot. Dog started showing visible recovery after two days and was recovered fully after ten days. Re-examination of blood after seven days for hematobiochemical parameters showed marked improvement with normal liver specific enzymes as well as total and direct bilirubin reached with in normal limits. 
Prehepatic icterus occurs when the rate of production of bilirubin exceeds the ability of the liver to excrete it. As the haemoglobin in red cells is the principle source of bilirubin, prehepatic icterus is seen with conditions where red cells are destroyed rapidly, thus invariably associated with moderate to severe anemia.

Ahmed et al., (2016) conducted a study on the etiology of anemia in 1749 dogs and different etiologies and their prevalence recorded were as Ehrlichiacanis (5.55\%), Babesia gibsoni (3.49\%), Immune mediated hemolytic anemia (IMHA) (1.49\%), Neoplasia (0.91\%), Babesia canis(0.51), Onion/Ginger toxicity (0.29\%) and others like non hemolyticanaemia were $(32.59 \%)$.

Boag, (2015) stated that, PCV estimationis always a prime concern in the icteric patients. If the animal is moderately or markedly anemic (approx. PCV $<25 \%$ in dogs), then the icterus is very likely to be prehepatic in origin and the underlying causes for haemolyticanaemia should be determined. Stabilization of the patient over the first few hours is likely to be empirical.

Fluid therapy should be based on an assessment of patient, whether the patient is hypovolaemic or dehydrated or both. Antibiotics or anti-parasitic agents needed for infectious or parasitic causes of hemolysis. In the present study, even the blood smear examination is negative for hemoprotozoan, imidocarb dipropionate was injected to the dog considering the possibility of missing the parasitemia in the blood smear.

Moreover, imidocarb dipropionate being broad spectrum antiprotozoal drug can also be used as prophylactic drug with minimum adverse effects. Chemoprophylactic approach with a few dated reports showed the effectiveness of imidocarb dipropionate against Babesia spp. Infection (Kuttler and Johnson, 1986; Vercammen et al., (1996). However, the chemoprophylactic use of these drugs should be restricted to immunosuppressed dogs (primarily splenectomised dogs) with an increased risk of exposure, including their residence in endemic areas (Irwin, 2009).

In the severe cases of anemia stabilization may include transfusion therapy, which allows time to identify the underlying cause of the anemia. In present study, haematological examination showed marked anaemia, decreased erythrocyte count and packed cell volume suggestive of immediate blood transfusion to save the life of the patient. Similar approach was followed by Perman and Schall (1983)where packed cell volume (PCV) was $15 \%$ or less and hemoglobin was $5 \mathrm{gm} \%$.

According to Godinho-Cunha et al., (2011) the most common indications for blood transfusion included acute blood loss (47\%), coagulopathy $(33 \%)$ and other anemias (20\%) and the mean pre-transfusion packed cell volume (PCV) of animals with acute blood loss $(18 \%)$ was higher than in the group of coagulopathy $(15 \%)$ or other anemias $(15 \%)$ which is similar to the present study indicating anemia because of other reasons. So, blood transfusion was done immediately for the survival of animal and to allow time for other medical treatment to work. Transfusion medicine is a lifesaving modality in case of emergency or critically ill animals. Post transfusion changes in the patient were similar to that of Kisielewicz et al., (2014) where haemoglobin concentration, PCV, and central venous oxygen $(\mathrm{CvO} 2)$ increased significantly with transfusion $(\mathrm{P}<.001)$ whereas lactate concentration decreased significantly $(\mathrm{P}=.006)$. 
Table.1 Hemato-biochemical findings of dog before and after blood transfusion

\begin{tabular}{|c|c|c|c|c|c|}
\hline \multicolumn{6}{|c|}{ Hematological parameters } \\
\hline S.No & Parameter & Day 0 & Day 7 & $\begin{array}{l}\text { Reference Range* } \\
\text { (The Merk } \\
\text { Veterinary manual) }\end{array}$ & Key findings \\
\hline 1 & $\mathrm{Hb}(\mathrm{g} \%)$ & 2.8 & 6.1 & $12-19$ & Anemia \\
\hline 2 & TEC (million/cmm) & 2.84 & 2.93 & $5-7.9$ & \\
\hline 3 & $\operatorname{PCV}(\%)$ & 12 & 20 & $37-55$ & \\
\hline 4 & $\mathrm{MCV}$ (fL) & 42.1 & 68.26 & $60-77$ & \multirow{3}{*}{$\begin{array}{l}\text { Microcytic } \\
\text { Hypochromic } \\
\text { Anemia }\end{array}$} \\
\hline 5 & $\mathrm{MCHC}(\mathrm{g} / \mathrm{dl})$ & 18.4 & 30.5 & $32-36$ & \\
\hline 6 & $\mathrm{MCH}(\mathrm{pg})$ & 17.7 & 20.81 & $19-25$ & \\
\hline 7 & TLC (thousand/cmm) & 29.17 & 18.9 & $5-14.1$ & \multirow{6}{*}{$\begin{array}{l}\text { Neutrophilic } \\
\text { leucocytosis }\end{array}$} \\
\hline 8 & Neutrophils (\%) & 88 & 79 & $58-85$ & \\
\hline 9 & Lymphocyte (\%) & 6 & 11 & $8-21$ & \\
\hline 10 & Monocyte (\%) & 4 & 7 & $2-10$ & \\
\hline 11 & Eosinophil (\%) & 2 & 1 & $0-9$ & \\
\hline 12 & Basophil (\%) & 0 & 0 & $0-1$ & \\
\hline 13 & Platelets(thousand/cmm) & 21 & 264 & $2.11-6.21$ & Normal \\
\hline \multicolumn{6}{|c|}{ Serum Biochemical Parameters } \\
\hline 14 & AST (IU/L) & 193.2 & 40.6 & $13-15$ & \multirow{2}{*}{$\begin{array}{c}\text { Increased liver } \\
\text { specific enzymes }\end{array}$} \\
\hline 15 & ALT (IU/L) & 243.1 & 66.7 & $10-109$ & \\
\hline 16 & Total Protein $(\mathrm{g} \%)$ & 4.26 & 4.26 & $5.5-7.5$ & \multirow{2}{*}{ Hypoalbuminemia } \\
\hline 17 & Albumin (mg\%) & 1.13 & 1.54 & $2.6-4.0$ & \\
\hline 18 & Total bilirubin (mg\%) & 2.76 & 0.44 & $0.1-0.6$ & \multirow{3}{*}{$\begin{array}{c}\text { Increased bilirubin } \\
\text { level }\end{array}$} \\
\hline 19 & Direct bilirubin $(\mathrm{mg} \%)$ & 1.24 & 0.23 & $0-0.4$ & \\
\hline 20 & Indirect bilirubin $(\mathrm{mg} \%)$ & 1.52 & 0.21 & $0-0.1$ & \\
\hline 21 & Serum creatinine $(\mathrm{mg} \%)$ & 1.1 & 0.73 & $0.5-1.7$ & $\begin{array}{l}\text { Normal serum } \\
\text { creatinine level }\end{array}$ \\
\hline
\end{tabular}


Table.2 Physiological parameters in every 20 min during Blood Transfusion in dog

\begin{tabular}{|c|c|c|c|c|c|c|c|c|c|c|c|c|c|c|c|}
\hline Parameter & \multicolumn{15}{|c|}{ Time interval (Minutes) } \\
\hline & T0 & $\mathrm{T} 20$ & $\mathrm{~T} 40$ & T60 & $\mathrm{T} 80$ & $\mathrm{~T} 100$ & $\mathrm{~T} 120$ & $\mathrm{~T} 140$ & $\mathrm{~T} 160$ & $\mathrm{~T} 180$ & T200 & $\mathrm{T} 220$ & $\mathrm{~T} 240$ & $\mathrm{~T} 260$ & $\mathrm{~T} 280$ \\
\hline $\begin{array}{c}\text { Temperature } \\
\left({ }^{0} \mathbf{F}\right)\end{array}$ & 97 & 99.8 & 98.9 & 97.5 & 97.8 & 97.1 & 98.2 & 97.3 & 97.8 & 98.2 & 97.1 & 97.5 & 96.9 & 97.5 & 97.8 \\
\hline $\begin{array}{c}\text { Heart rate } \\
\text { (Beats per } \\
\text { minute) }\end{array}$ & $\begin{array}{c}11 \\
7\end{array}$ & 110 & 114 & 108 & 109 & 109 & 106 & 105 & 104 & 107 & 108 & 107 & 107 & 109 & 109 \\
\hline $\begin{array}{c}\text { Pulse rate } \\
\text { (per minute) }\end{array}$ & 41 & 45 & 43 & 42 & 42 & 40 & 42 & 40 & 44 & 44 & 43 & 44 & 43 & 44 & 42 \\
\hline $\begin{array}{c}\text { Respiration } \\
\text { rate (per } \\
\text { minute) }\end{array}$ & 16 & 14 & 13 & 14 & 15 & 16 & 17 & 14 & 13 & 18 & 22 & 16 & 15 & 15 & 16 \\
\hline $\begin{array}{c}\mathrm{SpO2} \\
(\mathrm{mmHg})\end{array}$ & 65 & 94 & 69 & 68 & 68 & 69 & 66 & 69 & 66 & 66 & 68 & 78 & 71 & 84 & 80 \\
\hline
\end{tabular}

T0: Before transfusion; T20 $=20$ minutes after transfusion; Repeated-measures analysis of variance was calculated using two-way ANOVA for different parameters over the time and P value was calculated.

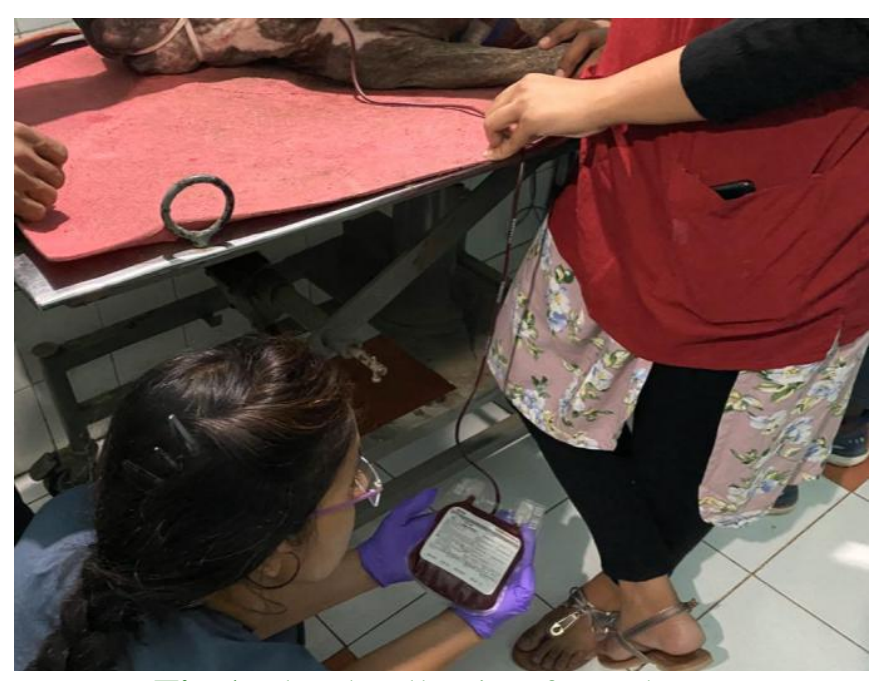

Fig.1 Blood collection from donor

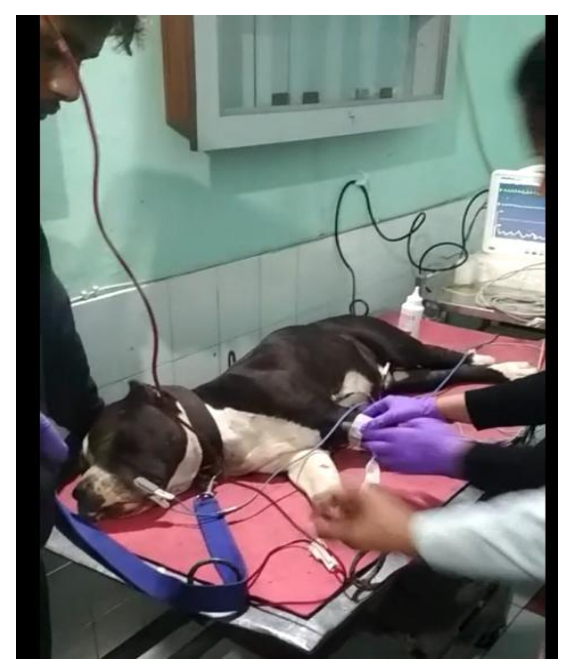

Fig.2 Blood transfusion in the recipient 
It is generally considered that the infusion of $20 \mathrm{ml} / \mathrm{kg}$ of whole blood volume is followed by increase in by $10 \%$ PCV (Kristensen and Feldman, 1995) which is comparable to our results. Patients should be appropriately screened with blood typing and cross matching before transfusion (Kumar, 2017; Ognean et al., 2015).

The appropriate use of transfusion medicine should balance the rare but not negligible potential risks associated with transfusions.

In the present study too, minor and major cross match was performed to ensure safe blood transfusion. No adverse reaction (immediate and delayed type) such as chills, allergic reactions characterized by urticaria, pruritus, erythema, flushing, hypotension and hemolysis, acute lung injury related to transfusion (TRALI), sepsis due to bacterial contamination, anaphylaxis, circulatory overload and metabolic complications were not reported in our study and the dog showed full recovery after transfusion.

Blood transfusion with proper match did not significantly change the physiological parameters like heart rate, temperature, pulse rate and respiration rate suggesting minimum interference with the normal body functioning.

Patients with acute anemias showed compromised oxygen-carrying capacity, representing different states of hypoxia or anoxia (Carson and Hébert 2009). In the present study too Spo2 was low before transfusion and reached to the desired limit after transfusion suggesting the reestablishment oxygen carrying capacity with normal pulse and heart rate.

This case report supports the use of whole blood transfusion in prehepatic jaundice with severe anemia of unknown causes.

\section{References}

Ahmed, B.R., Singh,D. P.,Kumar,S. N., Kumar,B. B., Kumar,U. S. 2016. Hospital Prevalence of Canine Hemolytic Anemia in Punjab. Journal of Animal Research. 6 (3):555-557.

Bhikane, A.U., and Kawitkar, S.B. 2002. Blood transfusion and fluid therapy. In: Hand book of veterinary clinicians. $1^{\text {st }}$ edn.Krishnapustakalaya, Latur. 325329.

Boag,A., 2015.The Icteric Patient. World Small Animal Veterinary Association World Congress Proceedings, 2015.

Carson,J.L and Hébert P. 2009. Anemia and Red BloodCell Transfusion. In: SIMON TL et al., (Eds), Rossi'sprinciples of transfusion medicine, Oxford: WileyBlackwell, p. 131-148.

Chakrabarti, A.,2006. Text book of clinical veterinary medicine. 3rd edn. Kalyani publishers, New Delhi 701.

Cotter, S. M., 1991. History of transfusion medicine. Advance in Veterinary Science and Comparitive Medicine. 36:1-8.

Davidow, B.,2013.Transfusion medicine in small animals.Veterinary Clinics of North America: Small Animal Practice. 43:735-756.

Godinho-Cunha, L. F., R. M. Ferreira and Silvestre-Ferreira A. C. 2011. Whole blood transfusion in small animals: indications and effects. Anais $d a$ Academia Brasileira de Ciências. 83(2): 611-617.

Harrell, K.A., and Kristensen, A. T. 1995. Canine transfusion reactions and their management. Veterinary Clinics of North America: Small Animal Practice.25: 1333-64.

Irwin, P.J., 2009. Canine babesiosis: from molecular taxonomy to control. Parasites \& vectors, 2(S1), p.S4.

Kisielewicz, C., Ian, A. S and Bell, R. 2014. 
Assessment of Clinical and Laboratory Variables as a guide to packed red blood cell transfusion of euvolemic anaemic dogs. Journal of Veterinary Internal Medicine.28: 576-82.

Kristensen, A.T., And Feldman, B. F. 1995. General principles of small animal blood component administration. Veterinary Clinic of North America: Small Animal Practice 25: 1277-1290.

Kumar, R.2017. Blood Transfusion in Veterinary Medicine. International Journal of Hematology and Transfusion. 4 (4): 116-122

Kuttler, K. L., and Johnson, L. W. 1986. Chemoprophylactic activity of imidocarb, diminazene and oxytetracycline againts Babesia bovis and B. Bigemina. Veterinary parasitology, 21(2): 107-118.

Lanevschi, A., and Wardrop, K. J. 2001. Principles of transfusion medicine in small animals. J. Canine Vet. 42: 447.

Lower, R.1665. The success of the experiment of transfusing the bloud of one animal into another. Philos Trans $\mathrm{R}$ Soc Lond B Biol Sci.1:352.

Ognean, L., V.Chiurciu, C. Ştefănuţ, L.Oana,
I. Morar and Barabási I. 2015. Transfusion triggers and therapeutic efficacy in a group of dogs that underwent whole blood therapy. Agriculture and Agricultural Science Procedia.6: 363-69.

Perman, V., and Schall, W. D. 1983. Diseases of the red blood cells. In: Text book of Veterinary Internal medicine. Diseases of the Dog and Cat edited by Ettinger, S. J.,2nd edn. W.B. Saunders Company, Philadelphia, pp. 1964 -1967.

Sevelius, E.,1995. Diagnosis and prognosis of chronic hepatitis and cirrhosis in dogs. Journal of Small Animal Practice, 36(12): 521-528.

Vercammen, F., R. De Deken and Maes, L. 1996. Prophylactic activity of imidocarb against experimental infection with Babesia canis. Veterinary parasitology. 63 (3-4) :195-198.

Weiss, D.J., and Wardrop, K.J. 2011. Schalm's veterinary hematology. John Wiley \& Sons.

Zuck, T.F.,1990. Legal liability for transfusion injury in the acquired immunodeficiency syndrome era. Arch Pathol Lab Med. 114: 309-15.

\section{How to cite this article:}

Tarun Kumar, Kanchan Arya, Pardeep Dhillon, Jai Bhagwan, Maneesh Sharma, Deepika Kumari, Divya Agnihotri and Jain. V. K. 2020. Successful Management of Jaundice in a Dog using Therapeutic Blood Transfusion. Int.J.Curr.Microbiol.App.Sci. 9(07): 600-608. doi: https://doi.org/10.20546/ijcmas.2020.907.067 